\title{
Observational Results of Full-Disc Helioseismology
}

\author{
Roger New \\ School of Science and Mathematics, Sheffield Hallam University, \\ Howard Street, Sheffield S1 $1 \mathrm{WB}, U K$
}

\begin{abstract}
Ever since the discovery of the low-degree p modes was announced by Claverie et al. (1979), full-disc helioseismology - seismology of the Sun based on the study of sunlight integrated across the entire disc - has contributed very significantly to our understanding of the deep solar interior and the origin and nature of the oscillation modes which probe it. This paper touches on the principal observational techniques used for full-disc helioseismology and outlines key results and their implications.
\end{abstract}

\section{Introduction}

In a Sun-as-a-star view, the amplitudes of oscillation modes with degree, $\ell$, greater than 4 average very nearly to zero. Hence the particular value of fulldisc helioseismic data is that they are optimized for the study of low- $\ell$ modes. Such modes are vital to inform our understanding of the Sun at depths below about $0.4 \mathrm{R}$, because the lower the degree of a $\mathrm{p}$ mode, the deeper in the Sun is its lower turning point. In addition to studies of the deep interior, full-disc data have been used to investigate the mode physics of low- $\ell$ oscillations and their solar cycle dependence, to investigate the excitation and damping mechanisms of the modes and to study the background velocity continuum. Finally, a full-disc view can inform the developing field of asteroseismology.

Historically the first extensive helioseismic data sets came from the full-disc ground-based networks, BiSON (Chaplin et al. 1996) and IRIS (Fossat 1995). Observations from the early 1990's are still routinely used in studies requiring extended data sets, for example to enhance mode detection, improve precision or to study solar cycle effects (for example, Chaplin et al. 2000; Salabert et al. 2003 ), and some studies can be performed with the less well-filled data from earlier epochs and from measurements from single sites.

\section{Techniques}

Full-disc helioseismic observations are carried out both in velocity and intensity, where the amplitude of the strongest $\mathrm{p}$ mode is of the order of $15 \mathrm{~cm} \mathrm{~s}^{-1}$ and a few ppm respectively. The small full-disc Doppler shift in a suitable Fraunhofer line can be determined by use of a resonant scattering spectrometer (RSS) (Grec at al. 1976, Brookes et al. 1978). In current operation, the instruments of the BiSON ground-based network (Chaplin et al. 1996) and the GOLF instrument on the ESA/NASA satellite SOHO (Gabriel et al. 1995) are different implementa- 


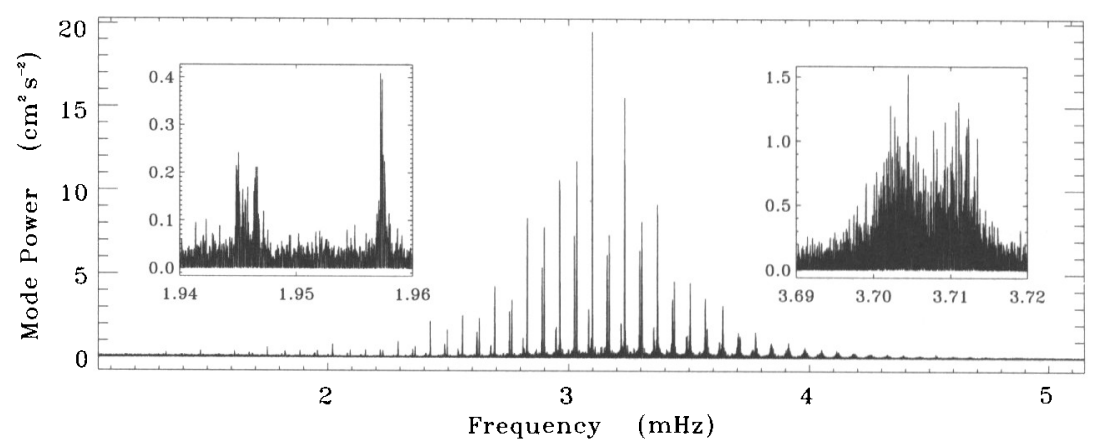

Figure 1. Low- $\ell$ p-mode spectrum of 3456 days of BiSON data. Each inset panel shows one $\ell=0$ and one $\ell=2$ mode. Rotational splitting of the low frequency $\ell=2$ mode is evident.

tions of the principles of the RSS. The intrinsic stability of the basic technique and careful instrument design means that modes with amplitudes lower than $10 \mathrm{~mm} \mathrm{~s}^{-1}$ can be observed in spectra of long data-sets.

Although extremely difficult from the ground due to the influence of the atmosphere, full-disc intensity measurements made by space-borne irradiance monitors have made a significant contribution to helioseismology. Pioneering observations were performed using the ACRIM instrument on the Solar Maximum Mission satellite (Woodard 1984), by IPHIR on PHOBOSII (Fröhlich et al. 1988), and the current VIRGO package on SOHO has full-disc (SPM) and low-resolution (LOI) intensity instruments (Fröhlich et al. 1995).

In addition to full-disc data, resolved-Sun low- $\ell$ results, such as those from MDI, GONG and ECHO have great value - as will be alluded to in Section 4 .

\section{The Low- $\ell$ Spectrum and the Deep Solar Interior}

Figure 1 illustrates two important features of the p-mode spectrum. Firstly, the mode amplitude falls off at both low and high frequency and, secondly, mode widths increase (by about 2 orders of magnitude) over the visible spectrum.

Near the center of the p-mode spectrum the frequency uncertainties can be as low as a few ppm and the intrinsic narrowness of the lower frequency modes allows their central frequencies to be determined to better than $10 \mathrm{nHz}$, even though the modes have low amplitudes (García et al. 2001; Chaplin et al. 2002a). More extended observation will improve this precision as the uncertainty scales as $T^{-1 / 2}$, where $T$ is the data-set length. The high precision already achieved makes the frequencies of the low- $\ell$ p modes a very effective probe of the deep interior. Although, for a given $\ell$, modes of higher frequency (and, therefore, higher radial order, $n$ ) penetrate deeper into the Sun, it is often the narrow, low- $n$, modes which can be used most easily to test models. See Goode (2001) for illustration and discussion.

Low-degree oscillation frequencies have been of great value in testing models of structure and dynamics of the deep interior. Firstly, well before the recent re- 
sults from the Sudbury Neutrino Observatory confirmed the existence of neutrino oscillations, the level of agreement between "standard" solar model predictions and observed low- $\ell$ p mode frequencies suggested that there would not be an "astrophysical" solution to the solar neutrino problem (see, for example, Chaplin et al 1997a). The agreement between calculated and observed frequencies is not, however, exact, and much theoretical effort is being focused on models with modified stratification near the core (Guzik et al 2000; Turck-Chièze et al. 2001; Gough 2003). For observers, the challenge is to produce ever more precise mode frequencies to better inform the model tests. This requires more extended observations and more detailed investigation of solar cycle variations.

Secondly, full-disc helioseismology has contributed significantly to understanding of the rotation of the deep interior, providing compelling evidence for a slowly (i.e. approximately surface rate) rotating core (see the review by Thompson et al. (2003)). The rotational splitting of the $\ell>0$ p modes (evident in the low frequency example in Figure 1) causes the individual $\ell+m$ even components (the only ones detectable in a full-disc view from Earth-orbit) to be separated by about $800 \mathrm{nHz}$. This is very much less than the line width of the modes at the higher frequency end of the spectrum. Great care is required to extract reliable estimates of rotational splittings from power spectra, and, again, low- $n$ modes are of great value because of their intrinsically small line width.

Two problems need to be flagged at this point. Firstly, p-mode parameters, including frequency, are functions of solar activity. It is becoming ever more important when using p-mode frequencies to test core models, and multiplet splittings to study rotation, that allowance is made for the activity level extant during the period of observation (Dziembowski \& Goode 1997). The phenomenology of solar cycle variations has been studied in detail in recent years (see Howe et al. (2003) and references therein) and cycle-dependent asymmetries have been reported in the $\ell=2$ multiplets used for rotation studies (Chaplin et al. 2003). Techniques for de-trending the data need further development.

Secondly, the peak profiles in the frequency spectrum are asymmetric. Fitting procedures have been developed which allow for, and determine the extent of, such asymmetries, returning more reliable estimates of the central frequencies of the modes, but recent comparisons between BiSON and GONG data show that the asymmetries are not yet fully understood (Howe et al. 2003).

\section{Mode Parameters and the Solar Cycle}

Although they can be determined with much poorer fractional uncertainty than central frequencies, there is also much important information to be gained from the widths and "strengths" of the p-mode profiles. Useful science can be obtained by studying peak heights over short time scales and by extracting peak heights and widths from analysis of power spectra of long time series.

The former analysis allows a test of the theory that $\mathrm{p}$ modes are generated by stochastic turbulence in the outer layers of the convection zone. Kumar et al. (1988) predicted that the distribution of mode powers, determined on a time scale short compared to the mode lifetimes, would follow negative exponential statistics. A number of studies (Chaplin et al. 1997b; Chang \& Gough 1998; 
Foglizzo et al. 1998) have shown this to be very largely the case, but longer data sets would help confirm the nature of a small number of extreme events.

More detailed information can be gained from the fitting of peak shapes to power spectra of long time series. Based on evidence that the modes are stochastically excited, fitting procedures generally employ maximum likelihood techniques to optimize the mode heights, widths, splittings, asymmetries and, where appropriate in ground based data, sideband leakage. Such analysis is not trivial and a wide range of studies have been carried out testing different strategies on real data, testing the data "pipelines" with simulated data and comparing results obtained from contemporaneous data from different instruments (for example, Thierry et al. 2001; Schou et al. 2002; Howe et al. 2003).

A number of points of consensus have been arrived at. Firstly, the variation of line width with frequency has been characterized and is in reasonable agreement with models of p-mode damping based on turbulent convection (Houdek et al. 2001). Secondly, the mode widths and powers are seen to change through the solar cycle. The widths increase by of the order of $20 \%$ near the center of the spectrum, while amplitudes fall by about $50 \%$ (Chaplin et al. 2000). To within the precision of present data, the width and power variations are consistent with increasing mode damping at high activity, while the energy supply rate from the stochastic source remains constant (Chaplin et al. 2002b; Jiménez et al. 2002).

\section{References}

Brookes, J. R., Isaak, G. R., \& van der Raay, H. B. 1978, MNRAS, 185, 1

Chang, H-Y., \& Gough, D. O. 1998, Solar Phys., 181, 251

Chaplin, W. J., Elsworth, Y., Isaak, G. R., Lines, R., McLeod, C. P., Miller, B. A., New, R., \& van der Raay, H. B. 1996, Observatory, 116, 32

Chaplin, W. J., Elsworth, Y., Isaak, G. R., McLeod, C. P., Miller, B. A., \& New, R. 1997a, ApJ, 480, L75

Chaplin, W. J., Elsworth, Y., Howe, R., Isaak, G. R., Lines, R., McLeod, C. P., Miller, B. A., \& New, R. 1997b, MNRAS, 287, 51

Chaplin, W. J., Elsworth, Y., Isaak, G. R., Miller, B. A., \& New, R. 2000, MNRAS, 313,32

Chaplin, W. J., Elsworth, Y., Isaak, G. R., Miller, B. A., \& New, R. 2002a, MNRAS, 330,731

Chaplin, W. J., Elsworth, Y., Isaak, G. R., Miller, B. A., New, R., \& Toutain, T. 2002b, ApJ, 582, L115

Chaplin, W. J., Elsworth, Y., Isaak, G. R., Miller, B. A., New, R., Thierry, S., Boumier, P., \& Gabriel, A. H. 2003, MNRAS, 343, 343

Claverie, A., Isaak, G. R., McLeod, C. P., van der Raay, H. B., \& Roca Cortés, T. 1979, Nature, 282, 591

Dziembowski, W. A., \& Goode, P. R. 1997, A\&A, 317, 919

Foglizzo, T., García, R. A., Boumier, P., Charra, J., Gabriel, A. H., Grec, G., Robillot, J-M., Roca Cortés, T., Turck-Chièze, S., \& Ulrich, R. K. 1998, A\&A, 330, 341

Fossat, E. 1995, in ASP Conf. Ser. Vol. 76, GONG'94: Helio- and Astero-Seismology, eds., R. K. Ulrich, \& E. J. Rhodes Jr., 387

Fröhlich, C., Bonnet, R. M., Bruns, A. V., Delaboudinière, J. P., Domingo, V., Kotov, V., Rashkovsky, D. N., Toutain, T., Vial, J. C., \& Wehrli, C. 1988, in ESA SP Vol.286, Seismology of the Sun and Sun-like Stars, ed. E. J. Rolfe, 359

Fröhlich, C., Romero, J., Roth, H., Wehrli, C., Andersen, N. B., Appourchaux, T., Domingo, V., Telljohann, U., Berthomieu, G., Delache, P., Provost, J., Toutain, T., Crommelnyk, D., Chevalier, D., Fichot, A., Däppen, W., Gough, D., Hoek- 
sema, T., Jimenéz, A., Gómez, M. F., Herreros, J., Roca Cortés, T., Jones, A. R., Pap, J., \& Willson, R. C. 1995, Solar Phys., 162, 101

Gabriel, A. H., Grec, G., Charra, J., Robillot, J-M., Roca Cortés, T., Turck-Chièze, S., Bocchia, R., Boumier, P., Cantin, M., Cespédes, E., Cougrand, B., Crétolle, J., Damé, L., Decaudin, M., Delache, P., Denis, N., Duc, R., Dzitko, H., Fossat, E., Fourmond, J. J., García, R., Gough, D., Grivel, C., Herreros, J. M., Lagardère, H., Moalíc, J. P., Pallé, P. L., Pétrou, N., Sanchez, M., Ulrich, R., \& van der Raay, H. B. 1995, Solar Phys., 162, 61

García, R., Régulo, C., Turck-Chièze, S., Bertello, L., Kosovichev, A. G., Brun, A. S., Couvidat, S., Henney, C. J., Lazrek, M., Ulrich, R. K., \& Varadi, F. 2001, Solar Phys., 200, 361

Goode, P. R. 2001, Solar Phys., 200, 343

Gough, D. O. 2003, Ap\&SS, 285, 341

Guzik, J. A., Neuforge-Verheeke, C., Young, A. C., Epstein, R. I., Poulin, F. M., \& Schissel, J. R. 2000, Solar Phys., 200, 305

Grec, G., Fossat, E., \& Vernin, J. 1976, A\&A, 50, 221

Houdek, G., Chaplin, W. J., Appourchaux, T., Christensen-Dalsgaard, J., Däppen, W., Elsworth, Y., Gough, D. O., Isaak, G. R., New, R., \& Rabello-Soares, M. C. 2001, MNRAS, 327, 483

Howe, R., Chaplin, W. J., Elsworth, Y., Hill, F., Komm, R., Isaak, G. R., \& New, R. 2003, MNRAS, 588, 1204

Jiménez, A., Roca Cortés, T., \& Jiménez-Reyes, S. J. 2002, Solar Phys.,209, 247

Kumar, P., Franklin, J., \& Goldreich, P. 1988, ApJ, 328, 879

Salabert, D., Jiménez-Reyes, S. J., \& Tomczyk, S. 2003,A\&A, 408,729

Schou, J., Howe, R., Basu, S., Christensen-Dalsgaard, J., Corbard, T., Hill, F., Komm, R., Larsen, R. M., Rabello-Soares, M. C., \& Thompson, M. J. 2002, ApJ, 567, 1234

Thierry, S., Boumier, P., Gabriel, A. H., \& GOLF team 2001, in ESA SP Vol. 464, SOHO10/GONG Workshop: Helio- and Asterosiesmology at the dawn of the Millennium, eds, A. Eff-Darwich, P. Palle, \& A. Wilson, 119

Thompson, M. J., Christensen-Dalsgaard, J., Miesch, M. S., \& Toomre, J. 2003, Annual Rev. Astron. Astrophys., 41, 599

Turck-Chièze, S., Nghiem, P., Couvidat, S., \& Turcotte, S. 2001, Solar Phys., 200, 323

Woodard, M., 1984, Nature, 309, 530 